\title{
Selección de proveedores priorizando criterios sostenibles para productos: un enfoque de AHP en compras públicas peruanas
}

Bernardo CORDERo TORres ${ }^{1}$

ReCIBIDO: 06/02/2019 AcePTADo: 11/03/2019

\begin{abstract}
RESUMEN
Este trabajo se enfoca en las compras públicas sostenibles que buscan aminorar impactos ambientales negativos y mejorar los impactos sociales positivos, las cuales, para funcionar, requieren de cambios significativos dentro de la realidad del mercado de un país. En el caso peruano, la legislación establece la obligación de que los criterios sostenibles integren las compras del Estado homologadas a las necesidades para la adjudicación de bienes y servicios. De esta forma, la problemática que pretende resolver esta investigación reside en la inobservancia técnica para verificar el cumplimiento de la reglamentación vigente en el procedimiento de selección de proveedores que consideren los criterios de sostenibilidad, para ello, sugerimos la aplicación del proceso analítico jerárquico (AHP) como alternativa heurística de solución. Este modelo de selección permite mejorar el procedimiento administrativo al incrementar el grado de objetividad y reducir sesgos cognitivos. Con el fin de demostrar ello, aplicaremos la verificación en el proceso analítico jerárquico en un caso de estudio de selección de proveedores de lámparas LED de una institución pública.
\end{abstract}

Palabras-claves: Modelo de selección; proveedores; proceso analítico jerárquico (AHP); compras públicas sostenibles.

\section{INTRODUCCIÓN}

En 1992, en la cumbre de las Naciones Unidas en Río de Janeiro, se reconoció internacionalmente el desafío del desarroIlo sostenible. Así, promulgada la Ley General del Ambiente N. ${ }^{\circ}$ 28611 (Ministerio del Ambiente, 2005), en el Perú, se introdujo la sostenibilidad como un principio en las compras públicas (CP), la cual es referida en los artículos 37, "asignación de puntajes especiales en licitaciones públicas a los proveedores ambientalmente más responsables" (p. 36), y 82.2, "definición de los puntajes de los procesos de selección de proveedores del Estado" (p. 52), donde se recomienda considerar el consumo racional y sostenible en las adquisiciones públicas.

A través del Decreto Supremo N. $184-2008-E F$, Reglamento de la Ley de Contrataciones del Estado (RLCE), no se llegó a esclarecer la aplicabilidad para la sostenibilidad en términos de ejecución de las compras públicas, tal como consta la ley N. ${ }^{\circ} 1017$ de Contrataciones del Estado, la cual menciona en su artículo 4, numeral $\mathrm{m}$, que en toda contratación "se aplicarán criterios para garantizar la sostenibilidad ambiental, procurando evitar impactos ambientales negativos en concordancia con las normas de la materia" (República del Perú, 2009, párr. 4).

En consecuencia a los preceptos de ley, el Ministerio del Ambiente (2016) difunde la ecoeficiencia para el sector público como estrategia para mejorar los servicios públicos en materia de desarrollo sostenible, ello bajo el Decreto Supremo N. ${ }^{\circ}$ 009-2009-MINAM que promueve la eficiencia en la erogación de fondos y minimiza los impactos negativos en el ambiente.

Por último, en 2017, el Decreto Supremo N. ${ }^{\circ} 350-2015-E F$ aprueba el RLCE modificado por el D. S. N. ${ }^{\circ} 056-2017-E F$, según la Ley de Contrataciones del Estado (LCE) N. ${ }^{\circ} 30225$, actualizada por el Decreto Legislativo N. ${ }^{\circ} 1341$, la cual establece que, en los procesos de selección, la sostenibilidad sea evaluada como un factor de decisión, de ahí que en su artículo 30, numeral b, se

1 Consultor independiente.

E-mail: consur2014@gmail.com

Magíster en Ingeniería Industrial con mención en Procesos Minero-metalúrgicos por la Universidad Andrés Bello. Es egresado de posgrado de la Facultad de Ingeniería Geológica, Minera, Metalúrgica y Geográfica de la Universidad Nacional Mayor de San Marcos. Lima, Perú ORCID: 0000-0001-9139-8672. 
señale que "las características particulares que se ofrecen para el objeto de contratación, como pueden ser las relacionadas a la sostenibilidad ambiental o social, mejoras para bienes y servicios, entre otras" (Kuczynski y Thorne, 19 de marzo de 2017, p. 12). A pesar de tipificarse la variable de sostenibilidad dentro del RLCE, persiste su inobservancia procedimental a nivel del comité de selección que permita tomar decisiones que no se vean condicionadas por inconsistencias en los juicios de los analistas, sea por ruido o por sesgo cognitivo (Kahneman, Rosenfield, Gandhi y Blaser, 2016).

El objetivo de esta investigación es aplicar el proceso analítico jerárquico (AHP), planteado por Saaty (1977), para desarrollar un análisis multicriterio más conocido, tal y como lo plantea Zanazzi (2003), el cual permita valorar las preferencias consistentes en relación con la forma de evaluación de factores del RLCE, respaldando al proceso decisorio como resultado de un método heurístico de evaluación de la mejor alternativa (Hertwig y Pachur, 2015). Se desea resaltar la funcionalidad que poseen las hojas de cálculo electrónicas disponibles en la web, las cuales no requieren la compra de licencias para uso de un software específico en la aplicación del AHP (Goepel, 2013).

Las limitaciones de este estudio recaen en el ámbito legal, al momento de formular factores técnicos de evaluación del objeto de la contratación que sean distintos a los que se encuadran en la ley; por lo tanto, hay un estrecho margen de maniobra para fijar criterios de sostenibilidad y establecer el seguimiento de los mismos. En todos los procesos de selección del Estado para la contratación de bienes o servicios, la oferta con menor precio es beneficiada, sin considerar sus "costos ambientales" (García, 2009). Se ha podido revisar, con relación a la temática, tratados puntuales sobre experiencias similares de compras públicas con criterios de sostenibilidad, exploradas por el TLC, el CAFTA, el Mercosur y la CAN (Beláustegui, 2011), y la iniciativa valor por dinero, en el caso peruano (Casier, Huizenga, Perera, Ruete y Turley, 2015).

\section{METODOLOGÍA}

\section{Establecimiento de la estrategia para evaluación de la sostenibilidad}

Para el estudio del caso, se adoptará a la ecoeficiencia (Ministerio del Ambiente, 2016) como estrategia para desarrollar una metodología de evaluación con criterios de sostenibilidad. Esta guía de ecoeficiencia está respaldada en la planificación plurianual del Perú, cuyo esfuerzo ha sido reconoci- do internacionalmente como una contribución hacia el desarrollo sostenible, según la Organización para la Cooperación y el Desarrollo Económicos (OCDE) y la Comisión Económica para América Latina y el Caribe (CEPAL) (2016), y el Ministerio Federal de Cooperación Económica y Desarrollo (BMZ) de Alemania (2016). Dicho desarrollo se extiende a todas las entidades del sector público y contempla cinco ejes temáticos principales:

1. Papel y materiales conexos.

2. Energía eléctrica y combustibles.

3. Agua.

4. Residuos sólidos.

\section{Cambio climático.}

\section{Evaluación según el enfoque del ciclo de vida} de los bienes y servicios

Evaluar la sostenibilidad en su contexto más amplio implica no solo revisar el marco social, ambiental o económico, sino también trascender lo netamente técnico y científico (Sala, Ciuffo y Nijkamp, 2015), ello considerando los alcances trazados en compromisos adquiridos internacionalmente mediante acuerdos que superan aspectos económicos y que generan procedimientos con criterios de selección transparentes en las compras gubernamentales, a favor de la sostenibilidad y del desarrollo humano (Arteaga, 2007; Navas, 2016). La metodología para ejecutar todos los procesos anteriores se respalda en la aplicación de la norma ISO 20400:2017, sistematizando la evaluación de la sostenibilidad bajo el enfoque del ciclo de vida (LCA) de los bienes y servicios, lo cual implica principiar en la etapa de la adquisición de la materia prima, o generación de recursos naturales, hasta su disposición final.

Cada categoría de los recursos debe estar alineada con los aspectos clave definidos por la estrategia de sostenibilidad de la organización, y que en el caso particular de las instituciones públicas considera la guía de ecoeficiencia (Ministerio del Ambiente, 2016). El alcance de la evaluación LCA de los bienes y servicios definidos por la organización tendrá en cuenta los propósitos y los recursos disponibles que podrían realizar estos tipos de análisis, ello con el fin de que se incluyan a los proveedores, contratos o prácticas organizacionales que influyan en las adquisiciones; asimismo, se considerarán a los criterios, como los costos totales, volúmenes de compra, utilización de los productos, entre otros, que mantengan vínculos significativos con los aspectos ambientales, sociales y económicos interactuantes 
entre sí, los cuales son interdependientes y denominados comúnmente como pilares de la sostenibilidad (International Organization for Standardization, 2017), como ilustra la Tabla 1.

\section{Integración de criterios sostenibles al proceso de selección}

Una vez fijadas las diferentes categorías de los impactos, dentro del enfoque del LCA de los bienes y servicios, frente al eje temático para el desarrollo de la estrategia de sostenibilidad, se definirán los criterios o subcriterios de selección de compra, a partir de los cuales, se contribuirá directamente a mejorar los impactos de los indicadores de desempeño de una forma más sostenible. Estos criterios tendrán que registrarse de manera documental, además, se deberá verificar que estos le sean aplicables a los bienes que se comprarán de forma directa. Algunos analistas podrán considerar asimismo otros parámetros, como los métodos de producción o los procesos utilizados para entregar bienes o servicios, y otros inherentes a la empresa proveedora.
Según la norma ISO 20400:2017, para incluir requisitos clave de sostenibilidad, que los criterios deben de cumplir durante el proceso de adquisiciones, estos deberán:

- Ser objetivos y verificables.

- Estar claramente definidos.

- Poder ser comunicados de forma transparente y eficaz a los posibles proveedores.

- Prestar atención especial a las mypes.

- Abarcar en la medida de las posibilidades toda la cadena de abastecimiento, permitiendo una evaluación de impacto adecuada.

- Incrementar el nivel de control en la cadena de abastecimiento.

- Definir los estándares mínimos, en caso sea aplicable, que los proveedores tendrán que cumplir desde los niveles más bajos de la cadena de abastecimiento.

Tabla 1. Evaluación de la ecoeficiencia versus la LCA de bienes y servicios.

\begin{tabular}{|c|c|c|c|c|c|}
\hline EJES TEMÁTICOS & $\begin{array}{l}\text { Extracción de } \\
\text { los recursos }\end{array}$ & Producción & Distribución & Uso & Disposición final \\
\hline Papel y materiales conexos & \multirow{5}{*}{$\begin{array}{l}\text { Impactos: } \\
\text { - Económico } \\
\text { - Ambiental } \\
\text { - Social }\end{array}$} & \multirow{5}{*}{$\begin{array}{l}\text { Impactos: } \\
\text { - Económico } \\
\text { - Ambiental } \\
\text { - Social }\end{array}$} & \multirow{5}{*}{$\begin{array}{l}\text { Impactos: } \\
\text { - Económico } \\
\text { - Ambiental } \\
\text {-Social }\end{array}$} & \multirow{5}{*}{$\begin{array}{l}\text { Impactos: } \\
\text { - Económico } \\
\text { - Ambiental } \\
\text { - Social }\end{array}$} & \multirow{5}{*}{$\begin{array}{l}\text { Impactos: } \\
\text { - Económico } \\
\text { - Ambiental } \\
\text { - Social }\end{array}$} \\
\hline Energía eléctrica y combustibles & & & & & \\
\hline Agua & & & & & \\
\hline Residuos sólidos & & & & & \\
\hline Cambio climático & & & & & \\
\hline
\end{tabular}

Fuente: Elaboración propia a partir de los datos obtenidos de Revilla (2017).

Tabla 2. Línea base de los ejes temáticos.

\begin{tabular}{|c|c|}
\hline Eje temático & Indicadores de desempeño \\
\hline \multirow{2}{*}{$\begin{array}{l}\text { Papel y materiales } \\
\text { conexos }\end{array}$} & Consumo de papel (millar o kg/colaborador/año) \\
\hline & Consumo de tintas/tóner (unidades/colaborador/año) \\
\hline \multirow{4}{*}{$\begin{array}{l}\text { Energía eléctrica y } \\
\text { combustibles }\end{array}$} & Consumo de energía eléctrica activa anual (kWh/colaborador/año) \\
\hline & Costo del consumo de energía eléctrica anual (costo/colaborador/año) \\
\hline & Consumo de energía (joule/año) \\
\hline & Costo de energía (costo/año) \\
\hline \multirow{2}{*}{ Agua } & Consumo de agua anual (m³/colaborador/año) \\
\hline & Costo del consumo de agua (costo/colaborador/año) \\
\hline Residuos sólidos & Generación de residuos reciclables, no reciclables y peligrosos (kg/colaborador/año) \\
\hline Cambio climático & $\begin{array}{l}\text { Generación anual de emisiones equivalentes de dióxido de carbono por colaborador ( } \mathrm{kg} \mathrm{CO}_{2 e q} / \text { colaborador/año) } \\
\text { Total anual de emisiones por colaborador }\left({\mathrm{t} \mathrm{CO}_{2} \mathrm{eq}}\right)\end{array}$ \\
\hline
\end{tabular}

Fuente: Elaboración a partir de los datos obtenidos de Ministerio del Ambiente (2016). 
Tabla 3. Aspectos de sostenibilidad a considerar en el proceso de selección de compras.

\begin{tabular}{|c|c|c|c|c|}
\hline Criterios & lad & Sub & verificación & CA \\
\hline $\begin{array}{c}\text { Sostenibilidad } \\
\text { ambiental }\end{array}$ & $\begin{array}{l}\text { - Alta } \\
\text { - Media } \\
\text { - Baja }\end{array}$ & $\begin{array}{l}\text { - Eficiencia energética } \\
\text { - Gestión de residuos } \\
\text { - Minimización de emisiones } \\
\text { - Desarrollo tecnológico } \\
\text { - Optimización de los recursos }\end{array}$ & $\begin{array}{l}\text { - Información técnica de los productos } \\
\text { - Certificaciones } \\
\text { - Etiquetas ecológicas } \\
\text { - Auditoría } \\
\text { - Documentación del proveedor } \\
\text { - Convenios para la disposición final de los } \\
\text { productos }\end{array}$ & $\begin{array}{l}\text { - Extracción de los } \\
\text { recursos } \\
\text { - Producción } \\
\text { - Distribución } \\
\text { - Uso } \\
\text { - Disposición final }\end{array}$ \\
\hline $\begin{array}{c}\text { Sostenibilidad } \\
\text { social }\end{array}$ & $\begin{array}{l}\text { - Alta } \\
\text { - Media } \\
\text { - Baja }\end{array}$ & $\begin{array}{l}\text { - Innovación social } \\
\text { - Número de beneficiarios } \\
\text { - Erradicación del trabajo infantil } \\
\text { - Contratación de personal con } \\
\text { discapacidad } \\
\text { - Seguridad y salud laboral } \\
\text { - Tipo de mype }\end{array}$ & $\begin{array}{l}\text { - Ficha técnica del producto } \\
\text { - Certificaciones } \\
\text { - Auditoría }\end{array}$ & $\begin{array}{l}\text { - Extracción de los } \\
\text { recursos } \\
\text { - Producción } \\
\text { - Distribución } \\
\text { - Uso } \\
\text { - Disposición final }\end{array}$ \\
\hline $\begin{array}{l}\text { Sostenibilidad } \\
\text { económica }\end{array}$ & $\begin{array}{l}\text { - Alta } \\
\text { - Media } \\
\text { - Baja }\end{array}$ & $\begin{array}{l}\text { - Garantía del fabricante } \\
\text { - Vida útil del producto } \\
\text { - Costo de mantenimiento } \\
\text { - Volúmenes de compra } \\
\text { - Razón del gasto de la compra y del } \\
\text { presupuesto anuales }\end{array}$ & $\begin{array}{l}\text { - Ficha técnica del producto } \\
\text { - Certificaciones } \\
\text { - Auditoría }\end{array}$ & $\begin{array}{l}\text { - Extracción de los } \\
\text { recursos } \\
\text { - Producción } \\
\text { - Distribución } \\
\text { - Uso } \\
\text { - Disposición final }\end{array}$ \\
\hline
\end{tabular}

Fuente: Elaboración propia a partir de los datos obtenidos de Revilla (2017).

Para la priorización de criterios de sostenibilidad en la presente investigación, se aplicará la información técnica obtenida de las fichas de homologación dispuesta en el D. S. N. ${ }^{\circ}$ 056-2017-EF. Por la ley LCE, PERÚ COMPRAS es el organismo encargado de publicar dichas fichas en su portal web. Asimismo, estas fichas son de cumplimiento obligatorio para las entidades contratantes, y su uso determinado para bienes y servicios permite delimitar que estos sean considerados recurrentes, estratégicos o masivos.

\section{AHP para priorización de los criterios de sostenibilidad}

El proceso de priorización AHP permite la toma de decisiones al tener múltiples criterios, ello mediante el empleo de variables cuantitativas y cualitativas (Salas, Leyva y Calenzani, 2014). Además, tiene diversas aplicaciones, desde permitir la selección de una vasta lista de proyectos de tecnologías de la información hasta la elección de editores de contenido educativo (Rivera, 2008; Huamaní y Eyzaguirre, 2015). En la Figura 1 se esquematizan los pasos secuenciales de dicho proceso.

\section{Representación gráfica de los resultados para seleccionar proveedores}

Al combinar los criterios de sostenibilidad, se conocerá el ranking con sus respectivos porcentajes de prioridades globales respecto a la meta global o problema de las ofertas de los proveedores, las cuales se graficarán en un sistema de referencia rectangular frente a la variable "precio", observando si existe o no una correlación lineal entre ambas variables (Saaty, 1990; Dias, Yamaguchi, Rabelo y Franco, 2012).

\section{RESULTADOS}

La formulación del requerimiento de la entidad contratante, detallada en la base estándar de adjudicación simplificada para la contratación de bienes, en la cual se basará el modelo de selección de proveedores, especifica el objetivo de seleccionar un proveedor de lámparas tipo $L E D$, correspondiéndole la mayor escala jerárquica, pues involucra al recurso de la energía eléctrica.

Del análisis de la evaluación de las recomendaciones del estudio de OCDE y CEPAL (2016), sobre el desempeño ambiental en el Perú, se recoge que tanto la sostenibilidad económica de las compras verdes, como la disposición final de residuos sólidos, cuentan con principal protagonismo y están consideradas dentro de las agendas de planificación del sector público. Este grupo de criterios se ubica en el nivel inmediatamente inferior al objetivo. 


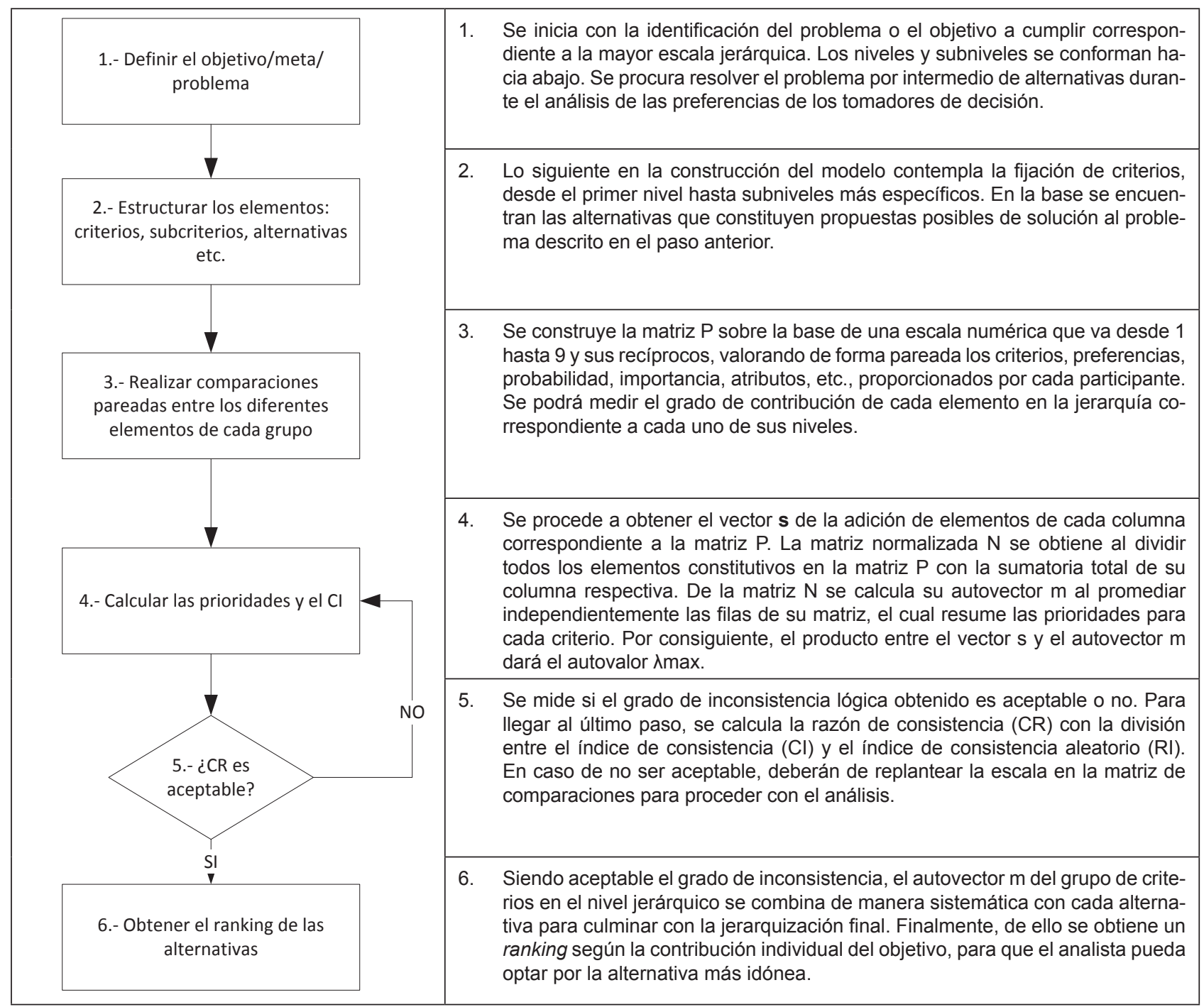

Figura 1. Pasos de la priorización AHP.

Fuente: Elaboración propia a partir de los datos obtenidos de Saaty (1977).

La información técnica de referencia para el análisis de los criterios, presentee en la Resolución Ministerial N. ${ }^{\circ}$ 494-2017-MEM/DM, aprueba 6 fichas de homologación para equipos de iluminación LED (ver Tabla 4), siendo per se la mejor opción frente a otras luminarias (Hernández, 2015). En la Figura 2 se muestra cómo los subcriterios de sostenibilidad siguen el orden jerárquico y culminan en las cuatro alternativas representadas por ofertas de los proveedores a evaluar.

El modelo de selección de proveedores, parametrizado en una hoja de cálculo, ingresa las mediciones de los decisores en la matriz $\mathrm{P}$, de la cual se desprende el vector sumatoria de cada columna $\vec{S}$ (ver Tabla 5) con su posterior cálculo de normalización en la matriz N (ver Tabla 6). Así, el insumo final arroja como resultante una priorización preliminar de los subcriterios representados como el autovector $\vec{m}$ y sus ponderaciones (ver Tabla 7 ).

Las prioridades preliminares de los subniveles están sujetas a comprobación, iniciando con el cálculo del autovalor $\lambda \max$ mediante la ecuación $\lambda \max =$ $\vec{s} \times \vec{m}$, donde $\lambda \max =6,602756957$.

Dado que $n$ equivale al número de subcriterios $(n=6)$, constituye un dato requerido para obtener el índice de consistencia (CI) mediante la expresión $C I=\frac{\lambda \max -n}{n-1}$, donde $C I=0,120551391$.

De otro lado, para evaluar si la razón de consistencia (CR) guarda consistencia lógica, se debe calcular la razón entre el índice de consistencia (CI) y el índice de consistencia aleatorio (RI), este último tomado de la Tabla 8 , y se aplica la fórmu- 
Tabla 4. Criterios y subcriterios de sostenibilidad para luminarias LED.

\begin{tabular}{|c|c|c|c|c|}
\hline Criterios & Prioridad & Subcriterios & Forma de verificación & Etapas del LCA \\
\hline $\begin{array}{l}\text { Sostenibilidad } \\
\text { ambiental } \\
\text { (SA) }\end{array}$ & $\begin{array}{l}\text { Media } \\
(\text { Del } 20 \% \text { al } 39 \%)\end{array}$ & $\begin{array}{l}\text { Eficiencia energética (EE): eficacia } \\
\text { luminosa } \\
\text { Disposición final (DF): convenio con } \\
\text { gestor de residuos }\end{array}$ & $\begin{array}{l}\text { Ficha técnica del producto } \\
\text { Fichas de homologación según R. M. N. } \\
\text { 494-2017-MEM/D } \\
\text { Declaración del proveedor }\end{array}$ & $\begin{array}{l}\text { Uso } \\
\text { Disposición final }\end{array}$ \\
\hline $\begin{array}{l}\text { Sostenibilidad } \\
\text { social } \\
\text { (SS) }\end{array}$ & $\begin{array}{l}\text { Baja } \\
\text { (Menor al 19\%) }\end{array}$ & $\begin{array}{l}\text { Confort visual (CV): temperatura de } \\
\text { color correlacionada K } \\
\text { Eficiencia del color (IC): índice de } \\
\text { rendimiento de color IRC }\end{array}$ & $\begin{array}{l}\text { Ficha técnica del producto } \\
\text { Fichas de homologación según R. M. N. } \\
\text { 494-2017-MEM/D }\end{array}$ & Uso \\
\hline $\begin{array}{l}\text { Sostenibilidad } \\
\text { económica } \\
\text { (SE) }\end{array}$ & $\begin{array}{l}\text { Alta } \\
\text { (Mayor al 40\%) }\end{array}$ & $\begin{array}{l}\text { Costo de mantenimiento (CM) } \\
\text { Vida útil de la luminaria LED (VU) }\end{array}$ & $\begin{array}{l}\text { Ficha técnica del producto } \\
\text { Fichas de homologación según R. M. N. }{ }^{\circ} \\
\text { 494-2017-MEM/D }\end{array}$ & Uso \\
\hline
\end{tabular}

Fuente: Elaboración propia.

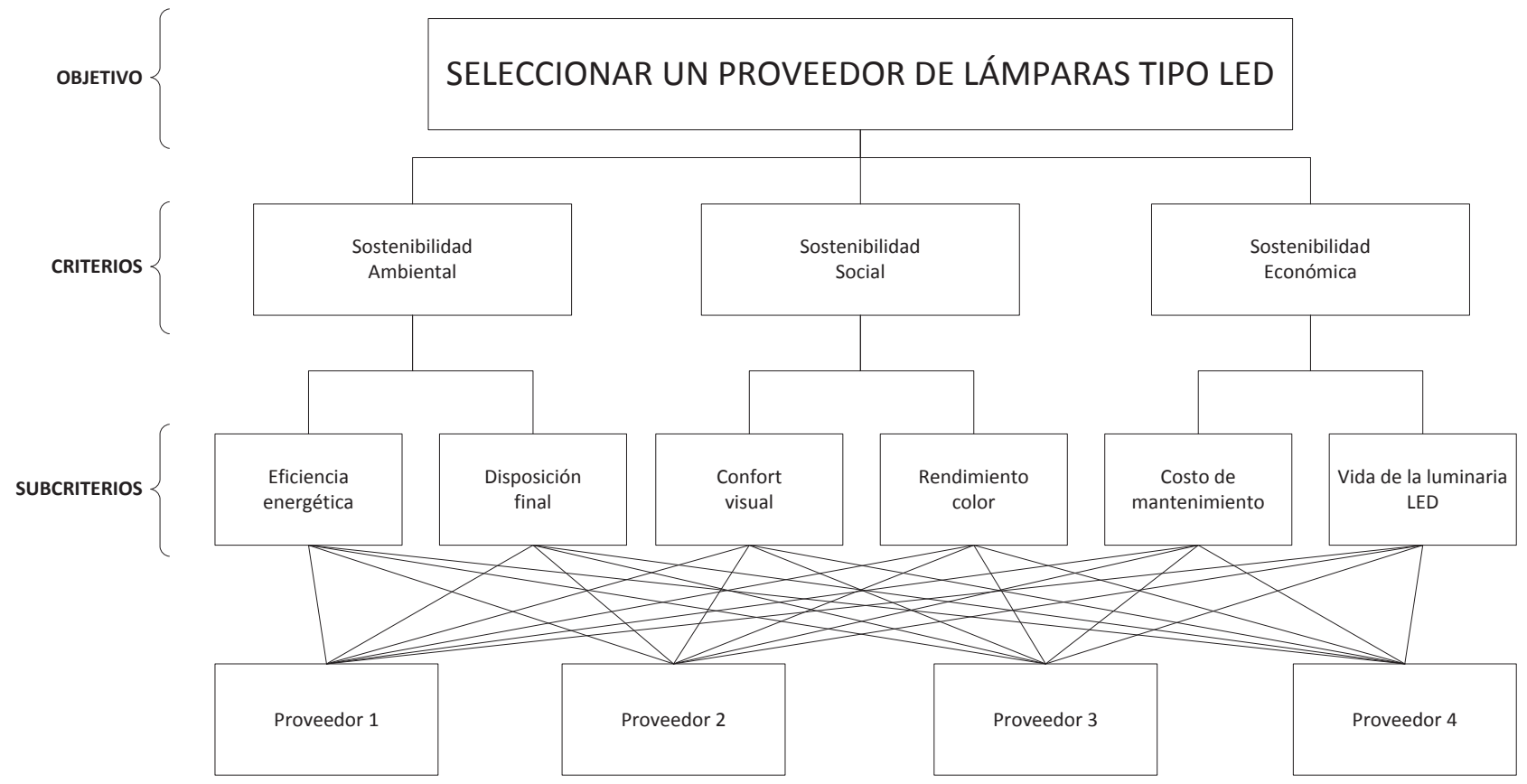

Figura 2. Estructura del modelo de selección.

Fuente: Elaboración propia a partir de los datos obtenidos de Saaty (1977).

Tabla 5. Ingreso de la matriz de comparaciones $P$ y cálculo del vector $\vec{s}$.

\begin{tabular}{|c|c|c|c|c|c|c|}
\hline & EE & DF & CV & RC & CM & VU \\
\hline EE & $\mathbf{1 , 0 0}$ & 0,50 & 3,00 & 4,00 & 1,00 & 2,00 \\
\hline DF & 2,00 & $\mathbf{1 , 0 0}$ & 2,00 & 3,00 & 0,50 & 0,33 \\
\hline CV & 0,33 & 0,50 & $\mathbf{1 , 0 0}$ & 0,50 & 0,20 & 0,14 \\
\hline RC & 0,25 & 0,33 & 2,00 & $\mathbf{1 , 0 0}$ & 0,17 & 0,14 \\
\hline CM & 1,00 & 2,00 & 5,00 & 6,00 & $\mathbf{1 , 0 0}$ & 1,00 \\
\hline VU & 0,50 & 3,00 & 7,00 & 7,00 & 1,00 & $\mathbf{1 , 0 0}$ \\
\hline Vector $\vec{S}$ & $\mathbf{5 , 0 8 3}$ & $\mathbf{7 , 3 3}$ & $\mathbf{2 0 , 0 0}$ & $\mathbf{2 1 , 5 0}$ & $\mathbf{3 , 8 7}$ & 4,62 \\
\hline
\end{tabular}

Fuente: Elaboración propia. 
Tabla 6. Matriz normalizada $N$.

\begin{tabular}{|c|c|c|c|c|c|c|}
\hline & EE & DF & CV & RC & CM & VU \\
\hline EE & 0,197 & 0,068 & 0,150 & 0,186 & 0,259 & 0,433 \\
\hline DF & 0,393 & 0,136 & 0,100 & 0,140 & 0,129 & 0,072 \\
\hline CV & 0,066 & 0,068 & 0,050 & 0,023 & 0,052 & 0,031 \\
\hline RC & 0,049 & 0,045 & 0,100 & 0,047 & 0,043 & 0,031 \\
\hline CM & 0,197 & 0,273 & 0,250 & 0,279 & 0,259 & 0,216 \\
\hline VU & 0,098 & 0,409 & 0,350 & 0,326 & 0,259 & 0,216 \\
\hline
\end{tabular}

Fuente: Elaboración propia.

Tabla 7. Autovector $\vec{m}$.

\begin{tabular}{|c|c|}
\hline Priorización de los criterios & Porcentaje \\
\hline 0,21542667 & 21 \\
\hline 0,161802739 & 16 \\
\hline 0,048277229 & 5 \\
\hline 0,052529631 & 5 \\
\hline 0,245605648 & 25 \\
\hline 0,276358083 & 28 \\
\hline
\end{tabular}

Fuente: Elaboración propia a partir de los datos obtenidos de Saaty (1977).

la $C R=\frac{C I}{R I} \times 100$, donde $C R=9,7$. La CR obtenida mantiene un grado aceptable, por cuanto se verifica que cumple la condición $C R<10$.

Tabla 8. Índice de consistencia aleatorio.

\begin{tabular}{|c|c|}
\hline $\boldsymbol{n}$ & $\mathbf{R I}$ \\
\hline 1 & 0 \\
\hline 2 & 0 \\
\hline 3 & 0,58 \\
\hline 4 & 0,9 \\
\hline 5 & 1,12 \\
\hline $\mathbf{6}$ & $\mathbf{1 , 2 4}$ \\
\hline 7 & 1,32 \\
\hline 8 & 1,41 \\
\hline 9 & 1,45 \\
\hline 10 & 1,49 \\
\hline
\end{tabular}

Fuente: Elaboración propia a partir de los datos obtenidos de Saaty (1990).

Siendo evaluada la $\mathrm{CR}$, las prioridades previamente calculadas (ver Tabla 9) permiten conocer el ranking de las ofertas de los proveedores, el cual es evaluado en la proximidad y el cumplimiento de cada uno de los subcriterios (ver Tabla 10).

Finalmente, la expresión gráfica de los resultados muestra que tanto el proveedor 2 como el provee- dor 3 poseen una relación precio y prioridad más atractiva, desde la perspectiva de la sostenibilidad (ver Figura 3), para ser favorecidos con la adjudicación del contrato.

\section{DISCUSIÓN}

En principio, se puede declarar que la compra de lámparas LED es más sostenible frente a luminarias del tipo fluorescente o incandescente (Hernández, 2015). Asimismo, es necesario profundizar en la información de las especificaciones técnicas del producto que los entes públicos vinculados disponen y que son aplicables a la realidad del Perú. De esta forma, una adecuada ejecución de la ecoeficiencia en el sector público permitirá condiciones favorables con cambios graduales en el mercado de oferta para desarrollar bienes de consumo más sostenibles.

El método por proceso analítico jerárquico (AHP) prioriza los criterios de sostenibilidad con base en comparaciones ordenadas, lo cual resulta idóneo para la selección de bienes y servicios, a pesar de ciertas limitaciones causadas por la disponibilidad de información y el grado de incertidumbre presentes en el proceso de adjudicación de contratos con proveedores en las compras públicas (Zanazzi, 2003). Así, la aplicación presentada del modelo AHP considera que las mediciones sobre las pre- 
Tabla 9. Priorización de los subcriterios.

\begin{tabular}{|c|c|c|c|c|c|}
\hline \multicolumn{2}{|c|}{ Subcriterio (\%) } & \multicolumn{2}{c|}{ Subcriterio (\%) } & \multicolumn{2}{c|}{ Subcriterio (\%) } \\
\hline EE & $21 \%$ & CV & $5 \%$ & CM & $25 \%$ \\
\hline DF & $16 \%$ & RC & $5 \%$ & VU & $28 \%$ \\
\hline
\end{tabular}

Fuente: Elaboración propia a partir de los datos obtenidos de Goepel (2013).

Tabla 10. Ranking de las ofertas de los proveedores.

\begin{tabular}{|c|c|c|}
\hline Número de ofertas & Prioridad (\%) & Precio (S/) \\
\hline Proveedor 1 & $84 \%$ & 82520 \\
\hline Proveedor 2 & $63 \%$ & 68500 \\
\hline Proveedor 3 & $53 \%$ & 63630 \\
\hline Proveedor 4 & $38 \%$ & 62940 \\
\hline
\end{tabular}

Fuente: Elaboración propia.

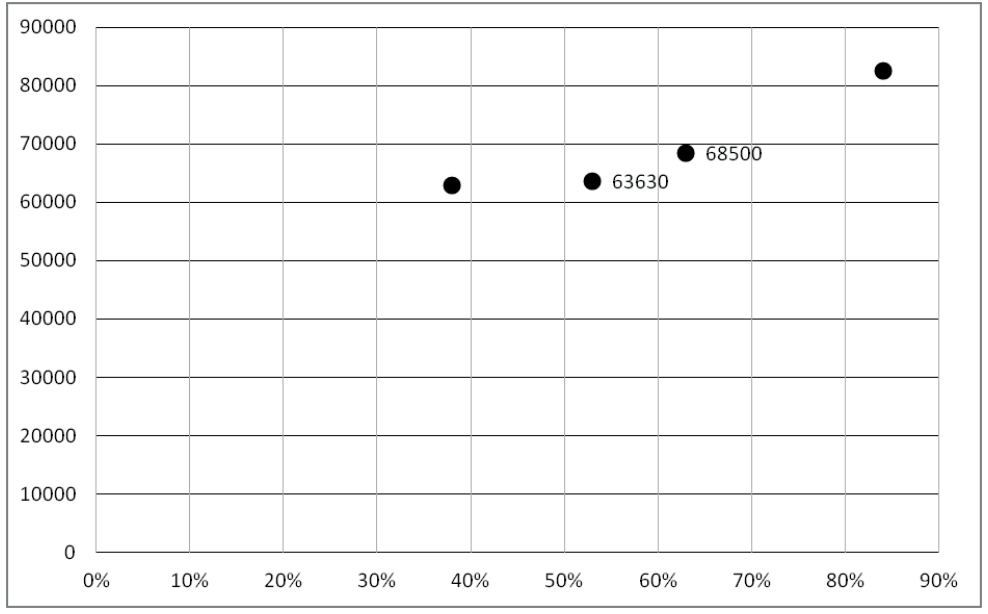

Figura 3. Ranking de las ofertas versus el precio.

Fuente: Elaboración propia.

ferencias son lineales y que el análisis se direcciona hacia el responsable de las adquisiciones de la entidad contratante, no involucrando a más participantes (Goepel, 2013). Como resultado, constituye un punto de partida para a posteriori extender el uso de criterios multidisciplinares sobre el ámbito de la sostenibilidad de forma más rigurosa.

Por otra parte, al ser un proceso de compras de naturaleza pública, existe dificultad en los grados de libertad para llevar a cabo un análisis de sensibilidad, lo que ata a cada oferente a un procedimiento administrativo, constriñendo el proceso si se lo compara con la cadena de suministros del sector privado. El mecanismo planteado de un proceso de selección con un análisis multicriterio busca instrumentar metodologías probadas acordes a requerimientos normativos nacionales e internacionales.

\section{CONCLUSIONES}

1. Al conseguir individualizar las especificaciones de productos que acarreen un mayor impacto positivo económico, ambiental y social a las entidades públicas, a estas les será posible mejorar las bases de las contrataciones, a fin de negociar mayores beneficios de las propuestas de los proveedores. También, se dará amplitud a la generación de procesos de medición y seguimiento de requisitos sostenibles, retroalimentando con información relevante a la rendición de cuentas de los fondos públicos.

2. Es deseable hacer notar la viabilidad técnica y económica de implementación del modelo AHP para la toma de decisiones extensiva al uso de herramientas informáticas del tipo open source 
o de libre acceso a los suscriptores, las cuales representan una ventaja relevante frente a las aplicaciones similares de software que requieren de licencia y conocimiento especializado.

3. Asimismo, cabe destacar que desde lo público es posible cumplir con las disposiciones legales y el apoyo del conocimiento científico, lo cual repercutirá favorablemente en las contrataciones públicas, ello mediante la reducción de los costos gracias a medios más ecoeficientes que contribuyen a la racionalización de los recursos y destinan el gasto de forma focalizada de acuerdo al presupuesto.

\section{REFERENCIAS BIBLIOGRÁFICAS}

[1] Arteaga, M. (2007). La Contratación Pública en el Marco del TLC Perú-EEUU. Derecho \& Sociedad, (29), 178-184.

[2] Beláustegui, V. (2011). Las compras públicas sustentables en América Latina. Estado de avance y elementos clave para su desarrollo. Buenos Aires, Argentina: Universidad Nacional de San Martín.

[3] Casier, L., Huizenga, R., Perera, O., Ruete, M. y Turley, L. (2015). Manual para agentes de compras públicas de la Red Interamericana de Compras Gubernamentales (RICG). Implementando Compras Públicas Sostenibles en América Latina y el Caribe. Optimizando el valor por dinero a través del ciclo de vida. Ginebra, Suiza: Instituto Internacional para el Desarrollo Sustentable (IISD).

[4] Dias, M., Yamaguchi, J., Rabelo, E. y Franco, C. (2012). Visualization Techniques: Which is the Most Appropriate in the Process of Knowledge Discovery in Data Base? En A. Karahoca (Ed.), Advances in Data Mining Knowledge Discovery and Applications (pp. 155-180). Rijeka, Croacia: InTech. Recuperado de https:// www.intechopen.com/books/advances-in-datamining-knowledge-discovery-and-applications/ visualization-techniques-which-is-the-mostappropriate-in-the-process-of-knowledgediscovery-in-data

[5] García, J. (2009). Las compras verdes, una práctica sustentable y ecológica: posibilidad de su aplicación en el Perú. Contabilidad y Negocios. Revista del Departamento Académico de Ciencias Administrativas, 4(7), 39-52.

[6] Goepel, K. (2013). Implementing the Analytic Hierarchy Process as a Standard Method for Multi-criteria Decision Making in Corporate
Enterprises-a new AHP Excel Template with Multiple Inputs. Recuperado de http://www. isahp.org/uploads/29.pdf

[7] Hernández, S. (2015). Análisis comparativo por ciclo de vida de tres tipos de luminarias empleadas en los interiores de edificios. Nova Scientia. Revista de Investigación de la Universidad De La Salle Bajío, 7(14), 538-559.

[8] Hertwig, R. y Pachur, T. (2015). Heuristics, History of. En International Encyclopedia of the Social \& Behavioral Sciences (pp. 829-835). Berlín, Alemania: Elsevier.

[9] Huamaní, G. T. y Eyzaguirre, R. (2015). Modelo de aplicación de AHP para seleccionar editor de contenidos de objetos de aprendizaje (modelo PAJOA-ECOA). Industrial Data. Revista de la Facultad de Ingeniería Industrial de la UNMSM, 18(2), 121-125.

[10] International Organization for Standardization (ISO) (2017). ISO 20100:2017. Sustainable procurement-Guidance. Ginebra, Suiza: ISO.

[11] Kahneman, D., Rosenfield, A. M., Gandhi, L., y Blaser, T. (2016). Noise: How to Overcome the High, Hidden Cost of Inconsistent Decision Making. Harvard business review, 94(10), 36-43.

[12] Kuczynski, P. P. y Thorne, A. (19 de marzo de 2017). Decreto Supremo que modifica el Reglamento de la Ley N. ${ }^{\circ} 30225$, Ley de Contrataciones del Estado, aprobado por el Decreto Supremo N. ${ }^{\circ}$ 350-2015-EF. El Peruano, pp. 5-50. Recuperado de http:// portal.osce.gob.pe/osce/sites/default/files/ Documentos/legislacion/ley/2017-Reg_ DL1341/DS-056-MODIFICACIONES\%20 AL\%20REGLAMENTO\%20LEY\%2030225.pdf

[13] Ministerio del Ambiente (2005). Ley General del Ambiente-Ley N. ${ }^{\circ}$ 28611. Recuperado de http://www.minam.gob.pe/wp-content/ uploads/2013/06/ley-general-del-ambiente.pdf

[14] Ministerio del Ambiente (2016). Guía de Ecoeficiencia para instituciones del Sector Público. Recuperado de http://sinia.minam. gob.pe/download/file/fid/59655

[15] Ministerio Federal de Cooperación Económica y Desarrollo (BMZ) de Alemania (2016). Alianza del Pacífico: Situación General de la Producción y el Consumo Sostenibles en Chile, Colombia, México y Perú. México D. F., México: Deutsche Geselischaft für Internationale Zusammenarbeit (GIZ). 
[16] Navas, C. (2016). Las contrataciones públicas: su trascendencia y desarrollo en el marco del comercio internacional y del TLC suscrito con Estados Unidos. Lex, 14(17), 259-275.

[17] Organización para la Cooperación y el Desarrollo Económicos (OCDE) y Comisión Económica para América Latina y el Caribe (CEPAL) (2016). Plan de acción para la implementación de las recomendaciones de la evaluación de desempeño ambiental. Lima, Perú: Ministerio del Ambiente.

[18] República del Perú (2009). Resolución de Secretaría General N. ${ }^{\circ}$ 062-2009-MINAM. Recuperado de http://www.minam.gob.pe/wpcontent/uploads/2013/09/directiva_sg_0062009-minam-sg.pdf

[19] Revilla, A. T. (2017). Acciones para impulsar las compras públicas ambientalmente sostenibles en el Perú. (Tesis de maestría). Pontificia Universidad Católica del Perú, Lima.

[20] Rivera, A. (2008). IT Project Portfolio Selection using Analytic Hierarchy Process. Industrial Data. Revista de la Facultad de Ingeniería Industrial de la UNMSM, 11(2), 59-62.

[21] Saaty, T. L. (1977). A Scaling Method for Priorities in Hierarchical Structures. Journal of Mathematical Psychology, 15(3), 234-281. Recuperado de https://doi.org/10.1016/00222496(77)90033-5

[22] Saaty, T. L. (1990). How to Make a Decision: The Analytic Hierarchy Process. European Journal of Operational Research, 48(1), 9-26. Recuperado de https://www.sciencedirect.com/science/ article/pii/037722179090057I?via\%3Dihub

[23] Sala, S., Ciuffo, B. y Nijkamp, P. (2015). A Systemic Framework for Sustainability Assessment. Ecological Economics, 119, 314-325. Recuperado de https://www. sciencedirect.com/science/article/pii/ S0921800915003821?via\%3Dihub

[24] Salas, J., Leyva, M. y Calenzani, A. (2014). Modelo del proceso jerárquico analítico para optimizar la localización de una planta industrial. Industrial Data. Revista de la Facultad de Ingeniería Industrial de la UNMSM, 17(2), 112-119.

[25] Zanazzi, J. L. (2003). Anomalías y supervivencia en el método de toma de decisiones de Saaty. En L. A. Godoy (Ed.), Problemas del Conocimiento en Ingeniería y Geología (volumen 1, pp. 148170). Córdoba, Argentina: Universitas. 


\section{Supplier selection prioritizing sustainable criteria for products: an AHP approach in Peruvian public procurement}

Bernardo CORDERo TORRES

\begin{abstract}
This paper focuses on sustainable public procurements that seek to minimize negative environmental impacts and improve positive social impacts, which require significant changes within the market reality of a country. In the case of Peru, legislation establishes the requirement that sustainable criteria be integrated into public procurement suited to the needs for the procurement of goods and services. In this way, the problem that this study seeks to resolve relies on technical non-observance to verify compliance with current regulations in force within the supplier selection process that consider sustainability criteria. Therefore, the application of the Hierarchy Analytic Process (AHP) is suggested as a heuristic solution alternative. This selection model makes it possible to improve the administrative process by increasing objectivity and reducing cognitive bias. To demonstrate this, the Analytic Hierarchy Process will be verified in a case study concerning the selection of LED lighting suppliers for a public institution.
\end{abstract}

Keywords: Selection model; suppliers; hierarchy analytical process (AHP); sustainable public procurement.

\section{INTRODUCTION}

In 1992, at the United Nations summit in Rio de Janeiro, the challenge that sustainable development represents was internationally recognized. Because of this, the Peruvian Ley General del Ambiente N. ${ }^{\circ} 28611$ [General Environmental Law No. 28611] (Ministerio del Ambiente, 2005) was enacted, and sustainability was introduced as a principle in public procurement (PP), which is referred to in section 37, "asignación de puntajes especiales en licitaciones públicas a los proveedores ambientalmente más responsables" [special scores assignment in public tenders to more environmentally-responsible suppliers] (p.36), and section 82, subsection 82.2, "definición de los puntajes de los procesos de selección de proveedores del Estado" [scoring definition of the selection processes for State suppliers] (p.52), where it is recommended to consider rational and sustainable consumption in public procurement.

The Supreme Decree No. 184-2008-EF, Reglamento de la Ley de Contrataciones del Estado [Regulation to the Government Procurement Law] (RLCE) did not clarify the applicability of sustainability in terms of public procurement fulfillment, as stated in Law No. 1017 of Government Procurement, which mentions in section 4, paragraph $\mathrm{m}$, that in every procurement "se aplicarán criterios para garantizar la sostenibilidad ambiental, procurando evitar impactos ambientales negativos en concordancia con las normas de la materia" [criteria to ensure environmental sustainability will be applied, while avoiding negative environmental impacts in accordance with relevant standards] (República del Perú, 2009, para. 4).

In consequence to the precepts of law, the Ministry of Environment (2016) promotes ecoefficiency in the public sector as a strategy to improve public services in sustainable development, under Supreme Decree No. 009-2009-MINAM, which promotes efficiency in the disbursement of funds and minimizes negative impacts on the environment.

\section{Independent consultant}

E-mail: consur2014@gmail.com

Master's in Industrial Engineering with a major in Mining-Metallurgical Processes at Universidad Andrés Bello. Postgraduate of the School of Geological, Mining, Metallurgical and Geographical Engineering of the Universidad Nacional Mayor de San Marcos. Lima, Peru ORCID: 0000-0001-9139-8672. 
Finally, in 2017, Supreme Decree No 350-2015EF approves the RLCE modified by Supreme Decree No. 056-2017-EF, in accordance with the Ley de Contrataciones del Estado No. 30225 [Government Procurement Law No. 30225] (LCE), amended by Legislative Decree No. 1341, which states that sustainability is evaluated as a decision factor in selection processes, which is why in section 30 , paragraph $b$, it is pointed out that "las características particulares que se ofrecen para el objeto de contratación, como pueden ser las relacionadas a la sostenibilidad ambiental o social, mejoras para bienes y servicios, entre otras" [the particular features offered for the procurement, such as those related to environmental and social sustainability, improvement for goods and services, among others] (Kuczynski \& Thorne, March 19, 2017, p.12) should be considered. Despite typifying the sustainability variable within the RLCE, its procedural non-compliance persists at selection committee level, which allow for decision making that appears to be conditioned by inconsistencies in the analysts' judgments, either by noise or by cognitive bias (Kahneman, Rosenfield, Gandhi \& Blaser, 2016). The aim of this study is to apply the hierarchy analytic process (AHP), posed by Saaty (1977), to develop a better known multicriteria analysis, as proposed by Zanazzi (2003), that will make it possible to assess consistent preferences in relation to RLCE factors evaluation, supporting a decision making process as a result of a heuristic evaluation for the best alternative (Hertwig \& Pachur, 2015). It is desired to highlight the functionality of electronic spreadsheets available on the web, which do not require the purchase of specific software licenses for AHP application (Goepel, 2013).

The limitations of this study fall within the legal sphere, when formulating technical factors of evaluation for the subject matter of the procurement that are different to those which fall within the law; therefore, there is little room for maneuver in order to set and follow-up on sustainability criteria. In all the public selection processes for contracting goods or services, the lowest price offer is preferred, without considering "environmental costs" (García, 2009). It has been possible to review, in relation to this subject, specific treaties on similar experiences of public procurement with sustainability criteria, explored by the FTA, CAFTA, Mercosur and the CAN (Beláustegui, 2011), and the value for money initiative, in the Peruvian case (Casier, Huizenga, Perera, Ruete \& Turley, 2015).

\section{METHODOLOGY}

\section{Establishment of the sustainability evaluation strategy}

For the case study, eco-efficiency will be adopted (Ministerio del Ambiente, 2016) as a strategy to develop an evaluation methodology with sustainability criteria. This ecoefficiency guide is supported in the multiannual budget plan of Peru, whose effort has been internationally recognized as a contribution towards sustainable development, according to the Organization for Economic Cooperation and Development (OECD) and the Economic Commission for Latin America and the Caribbean (ECLAC) (2016), and the Federal Ministry of Economic Cooperation and Development (BMZ) of Germany (2016). This development extends to all entities of the public sector and includes five main thematic pillars:

1. Paper and related materials.

2. Electric power and fuels.

3. Water.

4. Solid waste.

5. Climate change.

Evaluation according to the life cycle approach of goods and services

Evaluating sustainability in its broader context implies not only reviewing the social, environmental or economic framework, but also transcending what is purely technical and scientific (Sala, Ciuffo \& Nijkamp, 2015), considering the scope established by internationally acquired commitments through agreements that overcome economic aspects and generate procedures with transparent selection criteria in government procurements, in favor of sustainability and human development (Arteaga, 2007; Navas, 2016). The methodology for executing all the above processes is based on the application of the ISO 20400:2017 standard, systematizing the evaluation of sustainability under the life cycle approach (LCA) of goods and services, which implies beginning at the raw materials acquisition, or natural resources generation stage, until its final disposal. Each resource category should be aligned with the key aspects defined by the organization's sustainability strategy, and in the particular case of public institutions, consider the ecoefficiency guide (Ministerio del Ambiente, 2016). The scope of the LCA evaluation of goods and services defined by the organization will take into account the purposes and available resources that could make these 
types of analysis, in order to include suppliers, contracts or organizational practices that influence in procurements. Likewise, the criteria, such as total costs, purchase volumes, use of the products, among others, that maintain significant links with the interacting environmental, social and economic aspects, which are interdependent and commonly referred to as sustainability pillars (International Organization for Standardization, 2017), will be considered, as illustrated in Table 1.

\section{Integration of sustainable criteria into the selection process}

Once the different categories of impact have been established within the LCA approach of goods and services, in front of the thematic area for the development of the sustainability strategy, the criteria or sub-criteria for selection of purchase will be defined; these will directly contribute to improve the impact of performance indicators in a more sustainable way. These criteria will have to be recorded in documents, and it must be verified that these are appli- cable to the goods that will be purchased directly. Some analysts may also consider other parameters, such as production methods or processes used to deliver goods or services, and others inherent to the supplier company.

According to ISO 20400:2017 standard, to include key sustainability requirements, whose criteria must be met during the procurement process, these must:

- Be objective and verifiable.

- Be clearly defined.

- Be transparent and effective to be communicated to potential suppliers.

- Pay special attention to MSEs

- Cover as much as possible all the supply chain, allowing an adequate impact evaluation.

- Increase the level of control in the supply chain

Table 1. Evaluation of eco-efficiency versus LCA of goods and services.

\begin{tabular}{|c|c|c|c|c|c|}
\hline THEMATIC PILLARS & $\begin{array}{l}\text { Resources } \\
\text { extraction }\end{array}$ & Production & Distribution & Use & Final disposal \\
\hline Paper and related materials & \multirow{5}{*}{$\begin{array}{l}\text { Impact: } \\
\text { _Economic } \\
\text { _Environmental } \\
\text { _Social }\end{array}$} & \multirow{5}{*}{$\begin{array}{l}\text { Impact: } \\
\text {-Economic } \\
\text {-Environmental } \\
\text {-Social }\end{array}$} & \multirow{5}{*}{$\begin{array}{l}\text { Impact: } \\
\text {-Economic } \\
\text {-Environmental } \\
\text {-Social }\end{array}$} & \multirow{5}{*}{$\begin{array}{l}\text { Impact: } \\
\text {-Economic } \\
\text {-Environmental } \\
\text {-Social }\end{array}$} & \multirow{5}{*}{$\begin{array}{l}\text { Impact: } \\
\text {-Economic } \\
\text {-Environmental } \\
\text {-Social }\end{array}$} \\
\hline Electric power and fuels & & & & & \\
\hline Water & & & & & \\
\hline Solid waste & & & & & \\
\hline Climate change & & & & & \\
\hline
\end{tabular}

Source: Prepared by the author based on the data obtained from Revilla (2017).

Table 2. Baseline of the thematic pillars.

\begin{tabular}{|l|l|}
\hline Main pillar & Performance indicators \\
\hline $\begin{array}{l}\text { Paper and related } \\
\text { materials }\end{array}$ & $\begin{array}{l}\text { Paper consumption (thousands or kg/employee/year) } \\
\text { Ink/toner consumption (units/collaborator/year) }\end{array}$ \\
\hline $\begin{array}{l}\text { Electric power and } \\
\text { fuels }\end{array}$ & $\begin{array}{l}\text { Annual active electric power consumption (kWh/collaborator/year) } \\
\text { Cost of annual electricity consumption (cost/contributor/year) } \\
\text { Power consumption (joule/year) } \\
\text { Cost of energy (cost/year) }\end{array}$ \\
\hline Water & $\begin{array}{l}\text { Annual water consumption (m3/collaborator/year) } \\
\text { Cost of water consumption (cost/collaborator/year) }\end{array}$ \\
\hline Solid waste & Generation of recyclable, non-recyclable and hazardous waste (kg/worker/year) \\
\hline Climate change & $\begin{array}{l}\text { Annual generation of equivalent emissions of carbon dioxide per collaborator }\left(\mathrm{kg} \mathrm{CO} \mathrm{CO}_{2 \mathrm{eq}} / \mathrm{collaborator} / \mathrm{year}\right) \\
\text { Total annual emissions per employee (t CO } 2_{\text {eq }} \text { ) }\end{array}$ \\
\hline
\end{tabular}

Source: Elaboration based on data obtained from Ministerio del Ambiente (2016). 
Table 3. Aspects of sustainability to be considered in the procurement selection process.

\begin{tabular}{|c|c|c|c|c|}
\hline Criteria & Priority & Subcriteria & Verification means & LCA Stages \\
\hline $\begin{array}{l}\text { Environmental } \\
\text { sustainability }\end{array}$ & $\begin{array}{l}\text {-High } \\
\text {-Medium } \\
\text {-Low }\end{array}$ & $\begin{array}{l}\text {-Energy efficiency } \\
\text {-Waste management } \\
\text {-Minimization de emissions } \\
\text {-Technological development } \\
\text {-Optimization of resources }\end{array}$ & $\begin{array}{l}\text {-Technical information of the products } \\
\text {-Certifications } \\
\text {-Ecological labels } \\
\text {-Audit } \\
\text {-Supplier documentation } \\
\text {-Agreements for the final disposal of } \\
\text { products }\end{array}$ & $\begin{array}{l}\text {-Extraction of resources } \\
\text {-Production } \\
\text {-Distribution } \\
\text {-Use } \\
\text {-Final disposal }\end{array}$ \\
\hline $\begin{array}{l}\text { Social } \\
\text { sustainability }\end{array}$ & $\begin{array}{l}\text {-High } \\
\text {-Medium } \\
\text {-Low }\end{array}$ & $\begin{array}{l}\text {-Social innovation } \\
\text {-Number of beneficiaries } \\
\text {-Eradication of child labor } \\
\text {-Hiring personnel with disabilities } \\
\text {-Occupational health and safety } \\
\text {-Type of SME }\end{array}$ & $\begin{array}{l}\text {-Product data sheet } \\
\text {-Certifications } \\
\text {-Audit }\end{array}$ & $\begin{array}{l}\text {-Extraction of resources } \\
\text {-Production } \\
\text {-Distribution } \\
\text {-Use } \\
\text {-Final disposal }\end{array}$ \\
\hline $\begin{array}{l}\text { Economic } \\
\text { sustainability }\end{array}$ & $\begin{array}{l}\text {-High } \\
\text {-Medium } \\
\text {-Low }\end{array}$ & $\begin{array}{l}\text {-Manufacturer warranty } \\
\text {-Useful life of product } \\
\text {-Maintenance cost } \\
\text {-Purchase volumes } \\
\text {-Reason for purchasing expenses } \\
\text { and annual budget }\end{array}$ & $\begin{array}{l}\text {-Product data sheet } \\
\text {-Certifications } \\
\text {-Audit }\end{array}$ & $\begin{array}{l}\text {-Extraction of resources } \\
\text {-Production } \\
\text {-Distribution } \\
\text {-Use } \\
\text {-Final disposal }\end{array}$ \\
\hline
\end{tabular}

Source: Prepared by the author based on the data obtained from Revilla (2017).

- Define minimum standards, if applicable, that suppliers will have to meet from the lowest levels of the supply chain.

For the prioritization of sustainability criteria in this study, the technical information obtained from the Type Approval Certificates in accordance to the Supreme Decree N. ${ }^{\circ} 056-2017-E F$ will be applied. Through LCE law, PERU COMPRAS is the agency charged with publishing these certificates on their web portal. Additionally, these certificates are mandatory for contracting entities, and their specific use for goods and services makes it possible to define that these be considered recurring, strategic or widespread.

\section{AHP for the prioritization of sustainability criteria}

AHP allows for decision-making to have multiple criteria, via the use of quantitative and qualitative variables (Salas, Leyva \& Calenzani, 2014). In addition, it has various applications, from allowing for selection from an extensive list of information technology projects to the selection of editors of educational content (Rivera, 2008, Huamaní \& Eyzaguirre, 2015). In Figure 1 the sequential steps of said process are schematized.

\section{Graphical representation of the results to select suppliers}

After combining the sustainability criteria, the ranking will be determined, with their respective percentages of global priorities with respect to the overall goal or problem of the suppliers' offers, which will be graphed in a rectangular reference system against the variable "price", observing whether or not there is a linear correlation between both variables (Saaty, 1990; Dias, Yamaguchi, Rabelo \& Franco, 2012).

\section{RESULTS}

The formulation of the contracting entity's requirement -detailed in the simplified standard basis for procurement of goods- on which the supplier selection model will be based specifies the objective of selecting a provider of LED lighting, which corresponds to the largest hierarchical scale, because it involves the electrical energy resource.

From analysis of the evaluation of the OECD and ECLAC study recommendations (2016) concerning environmental performance in Peru, it is indicated that both the economic sustainability of green purchases and the final disposal of solid waste have 


\begin{tabular}{|c|c|c|}
\hline 1. Define the objective & & $\begin{array}{l}\text { Begins with the identification of the problem or the objective to be fulfilled corres- } \\
\text { ponding to the highest hierarchical scale. Levels and sublevels are in descen- } \\
\text { ding order. The problem is solved by means of alternatives during the analysis } \\
\text { of decision maker preferences. }\end{array}$ \\
\hline $\begin{array}{l}\text { 2. Structure the elements: criteria, } \\
\text { subcriteria, alternatives, etc. }\end{array}$ & \multicolumn{2}{|r|}{$\begin{array}{l}\text { 2. The following step in the construction of the model contemplates the setting } \\
\text { of criteria, from the first level to more specific sub-levels. The alternatives that } \\
\text { constitute possible solutions to the problem described in the previous step are } \\
\text { at the base. }\end{array}$} \\
\hline $\begin{array}{c}\text { 3. Make pairwise comparisons } \\
\text { between the different elements of } \\
\text { each group. }\end{array}$ & & $\begin{array}{l}\text { The } P \text { matrix is constructed based on a numerical scale from } 1 \text { to } 9 \text { and their } \\
\text { reciprocals, assessing in pairs the criteria, preferences, probability, importance, } \\
\text { attributes, etc., provided by each participant. The degree of contribution of each } \\
\text { element can be measured in the hierarchy corresponding to each of its levels. }\end{array}$ \\
\hline 4. Calculate the priorities and the $\mathrm{Cl}$ & 4. & $\begin{array}{l}\text { Next, the } S \text { Vector is obtained from the addition of the elements of each column } \\
\text { corresponding to the } \mathrm{P} \text { matrix. The matrix normal form } \mathrm{N} \text { is obtained by dividing } \\
\text { all the constituent elements in the } \mathrm{P} \text { matrix with the total summation of their } \\
\text { respective column. The eigenvector } \mathrm{m} \text { (of the matrix } \mathrm{N} \text { ) is calculated by indepen- } \\
\text { dently averaging the rows of its matrix. This summarizes the priorities for each } \\
\text { criterion. Therefore, the product of the vector } \mathrm{s} \text { and the eigenvector } \mathrm{m} \text { will give } \\
\text { the eigenvalue } \lambda \text { max. }\end{array}$ \\
\hline & 5. & $\begin{array}{l}\text { Measure the degree of logical inconsistency obtained is acceptable or not. To } \\
\text { reach the last step, the consistency ratio }(\mathrm{CR}) \text { is calculated with the division } \\
\text { between the consistency index }(\mathrm{Cl}) \text { and the random consistency index (RI). If it } \\
\text { is not acceptable, the scale in the pairwise comparison matrix must be reconsi- } \\
\text { dered to proceed with the analysis. }\end{array}$ \\
\hline $\begin{array}{l}\text { 6. Obtain the ranking of the } \\
\text { alternatives }\end{array}$ & & $\begin{array}{l}\text { With an acceptable degree of inconsistency, the eigenvector } m \text { of the group of } \\
\text { criteria in the hierarchical level is combined systematically with each alternative } \\
\text { to culminate with the final hierarchy. Finally, a ranking is obtained according to } \\
\text { the individual contribution of the objective, so that the analyst can choose the } \\
\text { most suitable alternative. }\end{array}$ \\
\hline
\end{tabular}

Figure 1. AHP Prioritization Steps.

Source: Prepared by the author based on the data obtained from Saaty (1977).

a main role and are considered within the planning agendas of the public sector. This group of criteria is located at the level immediately below the objective.

The technical reference information for the analysis of the criteria, present in Ministerial Resolution No. 494-2017-MEM/DM, approves 6 approval certificates for LED lighting equipment (see Table 4), being per se the best option in the face of other lighting options (Hernández, 2015). Figure 2 shows how the sustainability sub-criteria follow the hierarchical order and culminate in the four alternatives represented by offers from the suppliers to evaluate.

The supplier selection model, parameterized in a spreadsheet, contains the decision makers' measurements in the P-matrix, from which the summation vector of each column $\vec{S}$ is obtained (see Table
5) with its subsequent calculation of normalization in matrix $\mathrm{N}$ (see Table 6). Thus, the final input yields as a result a preliminary prioritization of the subcriteria represented as the eigenvector $\vec{m}$ and their weightings (see Table 7).

The preliminary priorities of the sublevels are subject to verification, starting with the calculation of the $\lambda$ max eigenvalue through the equation $\lambda \max =$ $\vec{s} \times \vec{m}$, where $\lambda \max =6.602756957$.

Since $n$ equals the number of sub-criteria $(n=6)$, it constitutes an piece of data required to obtain the consistency index $(\mathrm{Cl})$ through the expression $C I=\frac{\lambda \max -n}{n-1}$, where $C I=0.120551391$.

On the other hand, to evaluate if the consistency ratio $(\mathrm{CR})$ maintains logical consistency, the ratio 
Table 4. Criteria and sustainability subcriteria for $L E D$ lighting

\begin{tabular}{|l|l|l|l|l|}
\hline Criteria & Priority & Subcriteria & Forma de verification & LCA Stages \\
\hline $\begin{array}{l}\text { Environmental } \\
\text { Sustainability (EnS) }\end{array}$ & $\begin{array}{l}\text { Medium } \\
(20 \% \text { to } 39 \%)\end{array}$ & $\begin{array}{l}\text { Energy efficiency (EE): luminous } \\
\text { efficacy } \\
\text { Final disposal (FD): agreement with } \\
\text { waste manager }\end{array}$ & $\begin{array}{l}\text { Product data sheet } \\
\text { Type Approval Certificates accor- } \\
\text { ding to M. R. No 494-2017-MEM/D } \\
\text { Supplier declaration }\end{array}$ & $\begin{array}{l}\text { Use } \\
\text { Final disposal }\end{array}$ \\
\hline $\begin{array}{l}\text { Social } \\
\text { Sustainability (SS) }\end{array}$ & $\begin{array}{l}\text { Low } \\
\text { (Lower than 19\%) })\end{array}$ & $\begin{array}{l}\text { Visual comfort (VC): correlated color } \\
\text { Color efficiency (CE): Color Rendering } \\
\text { Index (CRI) }\end{array}$ & $\begin{array}{l}\text { Product data sheet } \\
\text { Type Approval Certificates accord- } \\
\text { ing to M. R. No 494-2017-MEM/D }\end{array}$ & Use \\
\hline $\begin{array}{l}\text { Economic } \\
\text { Sustainability (ECS) }\end{array}$ & $\begin{array}{l}\text { High } \\
\text { (Higher than 40\%) }\end{array}$ & $\begin{array}{l}\text { Maintenance cost (MC) } \\
\text { Useful life of LED lighting (L) }\end{array}$ & $\begin{array}{l}\text { Type Approval Certificates accord- } \\
\text { ing to M. R. No 494-2017-MEM/D }\end{array}$ & Use \\
\hline
\end{tabular}

Source: Prepared by the author.

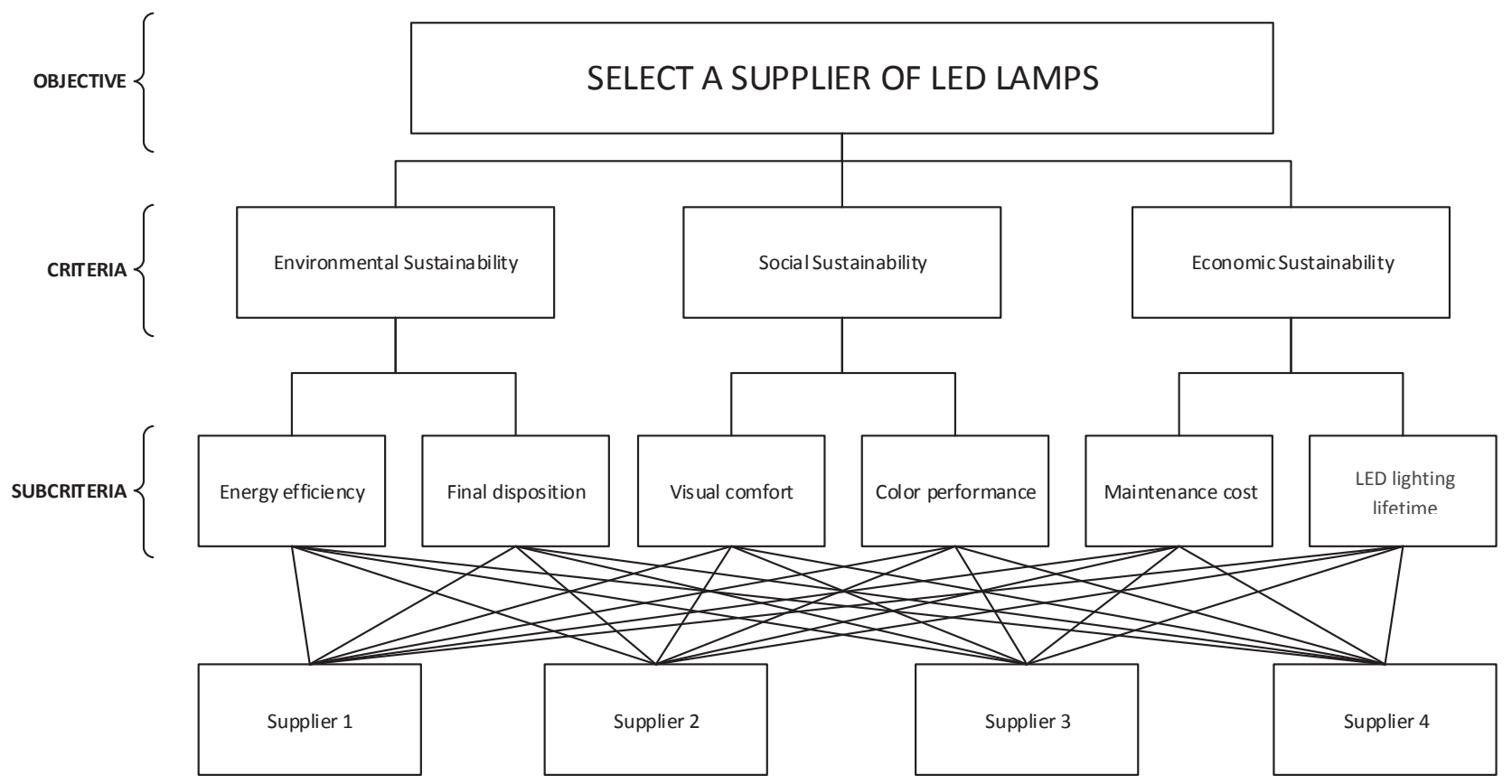

Figure 2. Structure of the selection model.

Source: Prepared by the author based on the data obtained from Saaty (1977).

Table 5. Input of the comparison matrix $P$ and calculation of the vector $\vec{S}$.

\begin{tabular}{|c|c|c|c|c|c|c|}
\hline & EE & FD & VC & CP & MC & L \\
\hline EE & $\mathbf{1 . 0 0}$ & 0.50 & 3.00 & 4.00 & 1.00 & 2.00 \\
\hline FD & 2.00 & $\mathbf{1 . 0 0}$ & 2.00 & 3.00 & 0.50 & 0.33 \\
\hline VC & 0.33 & 0.50 & $\mathbf{1 . 0 0}$ & 0.50 & 0.20 & 0.14 \\
\hline CP & 0.25 & 0.33 & 2.00 & $\mathbf{1 . 0 0}$ & 0.17 & 0.14 \\
\hline MC & 1.00 & 2.00 & 5.00 & 6.00 & $\mathbf{1 . 0 0}$ & 1.00 \\
\hline L & 0.50 & 3.00 & 7.00 & 7.00 & 1.00 & $\mathbf{1 . 0 0}$ \\
\hline Vector $\vec{S}$ & $\mathbf{5 . 0 8 3}$ & $\mathbf{7 . 3 3}$ & $\mathbf{2 0 . 0 0}$ & $\mathbf{2 1 . 5 0}$ & $\mathbf{3 . 8 7}$ & 4.62 \\
\hline
\end{tabular}

Source: Prepared by the author. 
Table 6. Normal matrix $N$

\begin{tabular}{|c|c|c|c|c|c|c|}
\hline & EE & FD & VC & CP & MC & L \\
\hline EE & 0.197 & 0.068 & 0.150 & 0.186 & 0.259 & 0.433 \\
\hline FD & 0.393 & 0.136 & 0.100 & 0.140 & 0.129 & 0.072 \\
\hline VC & 0.066 & 0.068 & 0.050 & 0.023 & 0.052 & 0.031 \\
\hline CP & 0.049 & 0.045 & 0.100 & 0.047 & 0.043 & 0.031 \\
\hline MC & 0.197 & 0.273 & 0.250 & 0.279 & 0.259 & 0.216 \\
\hline L & 0.098 & 0.409 & 0.350 & 0.326 & 0.259 & 0.216 \\
\hline
\end{tabular}

Source: Prepared by the author.

Tabla 7. Eigenvector $\vec{m}$.

\begin{tabular}{|c|c|}
\hline Prioritization of the criteria & Percentage \\
\hline 0.21542667 & 21 \\
\hline 0.161802739 & 16 \\
\hline 0.048277229 & 5 \\
\hline 0.052529631 & 5 \\
\hline 0.245605648 & 25 \\
\hline 0.276358083 & 28 \\
\hline
\end{tabular}

Source: Prepared by the author based on the data obtained from Saaty (1977).

between the consistency index (CI) and the random consistency index (RI) should be calculated, the latter taken from Table 8, by applying the formula $C R=\frac{C I}{R I} \times 100$, where $C R=9.7$. The CR obtained it is verified that it meets the condition $C R<10$.

Table 8. Random consistency index.

\begin{tabular}{|c|c|}
\hline $\boldsymbol{n}$ & $\mathbf{R I}$ \\
\hline 1 & 0 \\
\hline 2 & 0 \\
\hline 3 & 0,58 \\
\hline 4 & 0,9 \\
\hline 5 & 1,12 \\
\hline $\mathbf{8}$ & $\mathbf{1 , 2 4}$ \\
\hline 7 & 1,32 \\
\hline 8 & 1,41 \\
\hline 9 & 1,45 \\
\hline 10 & 1,49 \\
\hline
\end{tabular}

Source: Prepared by the author based on the data obtained from Saaty (1990).

When the CR is evaluated, the priorities previously calculated (see Table 9) allow the suppliers' offers ranking to be known, which is evaluated in the proximity and compliance of each subcriterion (see Table 10).
Finally, the graphical expression of the results shows that both supplier 2 and supplier 3 have a more attractive price and priority relationship, from the perspective of sustainability (see Figure 3 ), to be favored with the awarding of the contract.

\section{DISCUSSION}

In principle, it can be stated that the purchase of LED lighting is more sustainable than the purchase of fluorescent or incandescent lighting (Hernández, 2015). Likewise, it is necessary to perform a deep dive into the technical specifications of the product that are requested by public entities and which are suitable to conditions in Peru. In this way, an adequate ecoefficiency execution in the public sector will allow for favorable conditions with gradual changes in the supply market to develop more sustainable consumer goods.

The AHP method prioritizes sustainability criteria based on ordered comparisons, which is ideal for the selection of goods and services, despite certain limitations caused by the availability of information and the degree of uncertainty in the process of awarding contracts to suppliers in the public procurement market (Zanazzi, 2003). Thus, this application of the AHP model considers that the measurements on the preferences are linear and that the 
Table 9. Prioritization of subcriteria.

\begin{tabular}{|c|c|c|c|c|c|}
\hline \multicolumn{2}{|c|}{ Subcriterion (\%) } & \multicolumn{2}{c|}{ Subcriterion (\%) } & \multicolumn{2}{c|}{ Subcriterion (\%) } \\
\hline EE & $21 \%$ & CV & $5 \%$ & CM & $25 \%$ \\
\hline FD & $16 \%$ & RC & $5 \%$ & VU & $28 \%$ \\
\hline
\end{tabular}

Source: Prepared by the author based on the data obtained from Goepel (2013).

Table 10. Ranking of suppliers' offers.

\begin{tabular}{|c|c|c|}
\hline Number of offers & Priority (\%) & Price (S/) \\
\hline Supplier 1 & $84 \%$ & 82520 \\
\hline Supplier 2 & $63 \%$ & 68500 \\
\hline Supplier 3 & $53 \%$ & 63630 \\
\hline Supplier 4 & $38 \%$ & 62940 \\
\hline
\end{tabular}

Source: Prepared by the author.

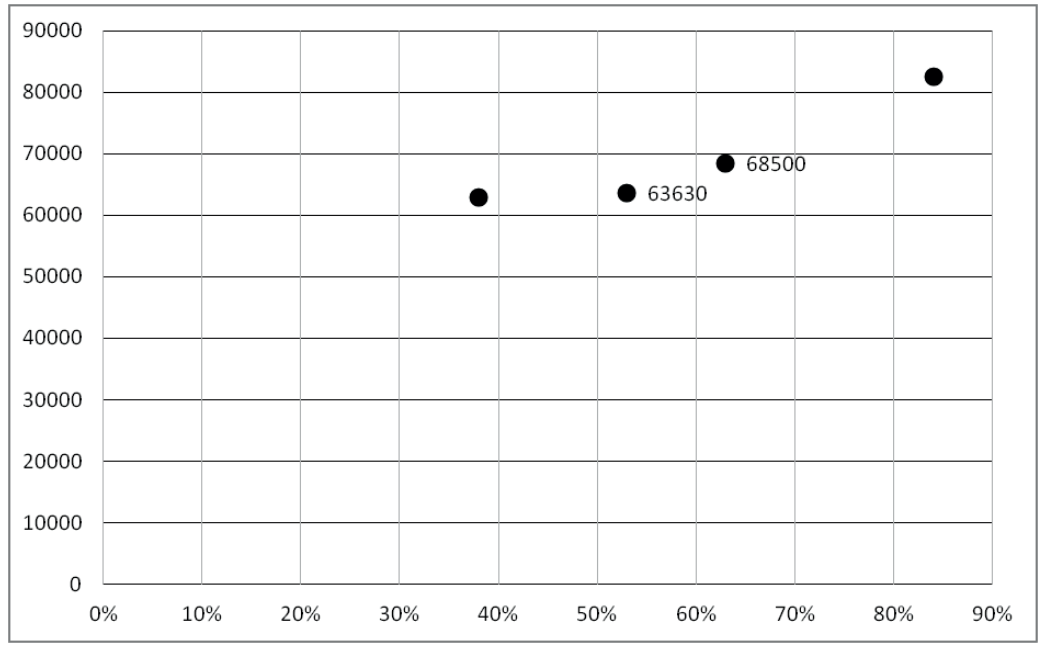

Figure 3. Ranking offers versus price.

Source: Prepared by the author.

analysis is directed towards the person responsible for the procurement of the contracting entity, not involving more participants (Goepel, 2013). As a result, it constitutes a starting point that a posteriori will extend the use of multidisciplinary criteria on the field of sustainability in a more rigorous way.

On the other hand, being a public procurement process, there is trouble in the degrees of freedom to carry out a sensitivity analysis, which binds each bidder to an administrative procedure, constraining the process if compared to the supply chain of the private sector. The proposed mechanism of a selection process with a multicriteria analysis seeks to implement proven methodologies according to national and international regulatory requirements.

\section{CONCLUSIONS}

1. By being able to individually identify product specifications that bring a greater positive economic, environmental and social impact to public entities, it will be possible to improve the bases of procurement, in order to negotiate greater benefits in supplier proposals. Also the processes for measuring and monitoring sustainable requirements will be extended, providing relevant information and feedback to the accountability of public funds.

2. It is desirable to point out the technical and economic feasibility for the AHP model for extensive decision-making to be applied using open source or free access tools, which represent a relevant 
advantage over similar software applications that require a license and specialized knowledge.

3. It should also be noted that from the public sector it is possible to comply with legal provisions and the help of scientific knowledge, which will have positive impacts on public procurement, via the reduction of costs through more ecoefficient means that contribute to wise use of resources and allocate spending in a focused manner in accordance to the budget.

\section{REFERENCES}

[1] Arteaga, M. (2007). La Contratación Pública en el Marco del TLC Perú-EEUU. Derecho \& Sociedad, (29), 178-184.

[2] Beláustegui, V. (2011). Las compras públicas sustentables en América Latina. Estado de avance y elementos clave para su desarrollo. Buenos Aires, Argentina: Universidad Nacional de San Martín.

[3] Casier, L., Huizenga, R., Perera, O., Ruete, M. \& Turley, L. (2015). Manual para agentes de compras públicas de la Red Interamericana de Compras Gubernamentales (RICG). Implementando Compras Públicas Sostenibles en América Latina y el Caribe. Optimizando el valor por dinero a través del ciclo de vida. Geneva, Switzerland: Instituto Internacional para el Desarrollo Sustentable (IISD).

[4] Dias, M., Yamaguchi, J., Rabelo, E. \& Franco, C. (2012). Visualization Techniques: Which is the Most Appropriate in the Process of Knowledge Discovery in Data Base? En A. Karahoca (Ed.), Advances in Data Mining Knowledge Discovery and Applications (pp. 155-180). Rijeka, Croatia: InTech. Retrieved from https:// www.intechopen.com/books/advances-in-datamining-knowledge-discovery-and-applications/ visualization-techniques-which-is-the-mostappropriate-in-the-process-of-knowledgediscovery-in-data

[5] García, J. (2009). Las compras verdes, una práctica sustentable y ecológica: posibilidad de su aplicación en el Perú. Contabilidad y Negocios. Revista del Departamento Académico de Ciencias Administrativas, 4(7), 39-52.

[6] Goepel, K. (2013). Implementing the Analytic Hierarchy Process as a Standard Method for Multi-criteria Decision Making in Corporate Enterprises-a new AHP Excel Template with
Multiple Inputs. Retrieved from http://www. isahp.org/uploads/29.pdf

[7] Hernández, S. (2015). Análisis comparativo por ciclo de vida de tres tipos de luminarias empleadas en los interiores de edificios. Nova Scientia. Revista de Investigación de la Universidad De La Salle Bajío, 7(14), 538-559.

[8] Hertwig, R. \& Pachur, T. (2015). Heuristics, History of. En International Encyclopedia of the Social \& Behavioral Sciences (pp. 829-835). Berlin, Germany: Elsevier.

[9] Huamaní, G. T. \& Eyzaguirre, R. (2015). Modelo de aplicación de AHP para seleccionar editor de contenidos de objetos de aprendizaje (modelo PAJOA-ECOA). Industrial Data. Revista de la Facultad de Ingeniería Industrial de la UNMSM, 18(2), 121-125.

[10] International Organization for Standardization (ISO) (2017). ISO 20100:2017. Sustainable procurementGuidance. Geneva, Switzerland: ISO.

[11] Kahneman, D., Rosenfield, A. M., Gandhi, L., \& Blaser, T. (2016). Noise: How to Overcome the High, Hidden Cost of Inconsistent Decision Making. Harvard business review, 94(10), 36-43.

[12] Kuczynski, P. P. \& Thorne, A. (2017, March 19). Decreto Supremo que modifica el Reglamento de la Ley N. ${ }^{\circ} 30225$, Ley de Contrataciones del Estado, aprobado por el Decreto Supremo N. ${ }^{\circ}$ 350-2015-EF. El Peruano, pp. 5-50. Retrieved from http://portal.osce.gob.pe/osce/sites/ default/files/Documentos/legislacion/ley/2017Reg_DL1341/DS-056-MODIFICACIONES\%20 AL\%20REGLAMENTO\%20LEY\%2030225.pdf

[13] Ministerio del Ambiente (2005). Ley General del Ambiente-Ley N. ${ }^{\circ}$ 28611. Retrieved from http://www.minam.gob.pe/wp-content/ uploads/2013/06/ley-general-del-ambiente.pdf

[14] Ministerio del Ambiente (2016). Guía de Ecoeficiencia para instituciones del Sector Público. Retrieved from http://sinia.minam.gob. pe/download/file/fid/59655

[15] Ministerio Federal de Cooperación Económica y Desarrollo (BMZ) de Alemania (2016). Alianza del Pacífico: Situación General de la Producción y el Consumo Sostenibles en Chile, Colombia, México y Perú. Mexico D. F., Mexico: Deutsche Geselischaft für Internationale Zusammenarbeit (GIZ).

[16] Navas, C. (2016). Las contrataciones públicas: su trascendencia y desarrollo en el marco del 
comercio internacional y del TLC suscrito con Estados Unidos. Lex, 14(17), 259-275.

[17] Organización para la Cooperación y el Desarrollo Económicos (OCDE) y Comisión Económica para América Latina y el Caribe (CEPAL) (2016). Plan de acción para la implementación de las recomendaciones de la evaluación de desempeño ambiental. Lima, Peru: Ministerio del Ambiente.

[18] República del Perú (2009). Resolución de Secretaría General N. ${ }^{\circ}$ 062-2009-MINAM. Retrieved from http://www.minam.gob.pe/wpcontent/uploads/2013/09/directiva_sg_0062009-minam-sg.pdf

[19] Revilla, A. T. (2017). Acciones para impulsar las compras públicas ambientalmente sostenibles en el Perú. (Tesis de maestría). Pontificia Universidad Católica del Perú, Lima.

[20] Rivera, A. (2008). IT Project Portfolio Selection using Analytic Hierarchy Process. Industrial Data. Revista de la Facultad de Ingeniería Industrial de la UNMSM, 11(2), 59-62.

[21] Saaty, T. L. (1977). A Scaling Method for Priorities in Hierarchical Structures. Journal of Mathematical Psychology, 15(3), 234-281. Retrieved from https://doi.org/10.1016/00222496(77)90033-5

[22] Saaty, T. L. (1990). How to Make a Decision: The Analytic Hierarchy Process. European Journal of Operational Research, 48(1), 9-26. Retrieved from https://www.sciencedirect.com/science/ article/pii/037722179090057I?via\%3Dihub

[23] Sala, S., Ciuffo, B. \& Nijkamp, P. (2015). A Systemic Framework for Sustainability Assessment. Ecological Economics, 119, 314-325. Retrieved from https://www. sciencedirect.com/science/article/pii/ S0921800915003821?via\%3Dihub

[24] Salas, J., Leyva, M. \& Calenzani, A. (2014). Modelo del proceso jerárquico analítico para optimizar la localización de una planta industrial. Industrial Data. Revista de la Facultad de Ingeniería Industrial de la UNMSM, 17(2), 112-119.

[25] Zanazzi, J. L. (2003). Anomalías y supervivencia en el método de toma de decisiones de Saaty. En L. A. Godoy (Ed.), Problemas del Conocimiento en Ingeniería y Geología (volumen 1, pp. 148170). Córdoba, Argentina: Universitas. 